\title{
Interleukin-12 and protective immunity to schistosomes
}

A.P. Mountford,
V.L. Shires and
S. Anderson

Correspondence

A.P. Mountford

Department of Biology

The University of York

P.O. Box 373

York YO1 5YW

UK

Fax: (44) 1904432884

E-mail: apm10@unix.york.ac.uk

Presented at the International

Meeting on Cytokines, Angra dos

Reis, RJ, Brasil, November 24-28,

1996.

Research supported by a Wellcome

Trust Career Development

Fellowship to A.P. Mountford;

S. Anderson was also supported by

this grant. V.L. Shires was the

recipient of a BBSRC fellowship.

...................

Received September 4, 1997

Accepted September 22, 1997

......................
Department of Biology, The University of York, York, UK

\section{Abstract}

The attenuated vaccine against Schistosoma mansoni induces Th1$m$ ediated protective i $m m$ unity and we have sought t $o$ identify a role for IL-12 in this model. Elevated levels of IL-12 (p40 $m$ RNA) were detected in the lymph nodes (LN) and the lungs of vaccinated mice, whilst treatment of vaccinated $m$ ice with anti-IL-12 antibodies decreased the ratio of IFN $\gamma$ :IL-4 secreted by in vitro-cultured LN cells. However, there was only $m$ arginal abrogation of the level of resistance in these mice. Soluble antigens from the lung-stage of the parasite (SLAP) appeared to be efficient stimulators of IFN $\gamma$ and IL-12 secretion. These antigens when used to immunise $m$ ice in conjunction with IL-12 as an adjuvant, elicited a polarised Th1 response with abundant IFN $\gamma$ secretion but no IL-4. This immunisation regime also induced significant protection against reinfection, whereas inoculation of $m$ ice with SLAP alone did not. The induction of a dominant Th1 response using SLAP + IL-12 probably operates via IFN $\gamma$ production by natural killer (NK) cells stimulated by IL-12, sincein vivo ablation of NK cells using anti-NK1.1 antibody reduced CD4+-dependent IFN $\gamma$ production from cultured LN cells by over $97 \%$. Nevertheless, in $m$ ice with a genetic disruption of the IFN $\gamma$ receptor, administration of SLAP + IL-12 induced levels of IFN $\gamma$ equal to those in wild-type $m$ ice, thus showing that in this model IL-12 can directly prime T cells independent of IFN $\gamma$. Clearly, IL-12 has a critical role in protective immunity to schistosomes and it $m$ ay aid the development of an effective vaccine against this disease.

\section{Introduction}

The recently identified cytokine interleukin-12 (IL-12) has a very important role during the early phases of an immune reaction, and it is a major factor in the differentiation of Thelper lymphocyte subsets. Thus, $m$ any researchers have studied its role in shaping the polarized phenotype of immune responses induced by various parasite infections. In this review, we will focus on the
Key words

- Schistosoma

- Interleukin-12

- Adjuvant

- Vaccine

- Th1 role of IL-12 in the induction of protective immunity to infection with the parasitic hel$m$ inth Schistosoma mansoni. Such studies give hope that IL-12 can aid the develop$m$ ent of an effective vaccine against this disease.

\section{Background to interleukin-12}

There are several excellent reviews on the biology of IL-12 and the reader is en- 
couraged to look to those by Wolf et al. (1) and Trinchieri (2) for detailed information. In brief, IL-12 is a heterodimeric molecule of approximately $70 \mathrm{kDa}$ composed of 2 glycoproteins of $40 \mathrm{kDa}(\mathrm{p} 40)$ and $35 \mathrm{kDa}$ (p35) and was originally termed natural killer cell stimulatory factor or cytotoxic lymphocyte maturation factor. The p35 chain appears to be constitutively expressed by $m$ any cell types, whereas the $\mathrm{p} 40$ chain and the biologically active p70 dimer are highly inducible and are produced by monocytes and dendritic cells. The ability of such cells to produce IL-12 whilst also acting as antigenpresenting cells (APCs) may be critical in driving the direction or polarisation of the immune response. The key role of IL-12 in vivo is its potent ability to induce IFN $\gamma$ production by both natural killer (NK) and $\mathrm{T}$ cells. It also works in synergy with other cytokines to enhance IFN $\gamma$ production (e.g., IL-2 and TNF $\alpha$ ) and is dependent upon other factors such as costimulatory interactions (i.e., CD28-B7). In contrast, IL-10, TGFß, IL-13 and IL-4 all have inhibitory effects on the production of IL- 12 .

The role of IL-12 in parasitic disease

Resistance to reinfection with intracellular parasites is frequently associated with dominant Th1 responses implying a role for IL-12. For example, susceptible mice infected with Leishmania major and treated with exogenous IL-12 mount a Th1 response and become resistant to reinfection $(3,4)$. In contrast, genetically resistant $m$ ice deficient for either the p35 or p40 subunits are susceptible to infection with L. major and mount a polarised Th2 cell response (5). Where NK cell activity has been implicated as an effective anti-parasite $m$ echanis $m$, in Toxoplasma gondii infections for example, then early ablation of IL-12 leads to chronic disease and a loss of the immune status (6), whilst ablation of IL-12 in resistant strains of $m$ ice infected with L. major leads to the develop- ment of Th2 responses and progression to disease $(3,4)$.

In general, it is thought that helminth infections induce protective Th2 responses leading to elevated seru $m$ IgE levels, eosinophilia, and mastocytosis. As expected, administration of IL-12 to mice infected with either Nippostrongylus brasiliensis (7) or Brugia malayi (8) promotes the switch in development from a biased Th2 response to one where Th1 responses dominate but this does not enhance host protection. The dominant Th2 responses to helminth infections are often associated with severe tissue pathology (e.g., the granulomatous response to schistosome eggs) and so IL-12 treatment $m$ ay be useful in downregulating such responses. In this respect, Wynn et al. (9) demonstrated that although sensitisation of $m$ ice with schistosome eggs and IL-12 did not reduce the number of adult worms or eggs recovered following challenge with a normal percutaneous infection, the granulo$m$ as around the challenge eggs were smaller, contained fewer eosinophils and had reduced hepatic fibrosis. However, there is also strong evidence that Th1 responses can be protective against the larval forms of schistosomes and so IL-12 $m$ ay have a critical role to play in the generation of protective immunity.

Th1 lymphocyte-mediated immunity toSchistosoma mansoni

Mice infected with normal S. mansoni clearly develop an immune response which is dominated by Th2 cells stimulated by the onset of egg deposition (10), although it remains unclear whether the response is host protective (11). In contrast, optimally attenuated schistosomes, which fail to migrate further than the lungs and do not $m$ ature, induce demonstrable high levels of protection $(60-70 \%)$ to challenge infection. Initially, a mixed Th1/Th2 or Th0 response is observed in cells from the draining lymph nodes (LN). However, by 7 days after vacci- 
nation a polarised Th1 phenotype has developed in the LN, whereby cells secrete abundant IFN $\gamma$ but $m$ inimal IL-4 (12). In contrast, normal larvae fail to sustain IFN $\gamma$ secretion in these LN after the first week. Antigenspecific cells of the Th1 subset are also recruited to, and persist for at least 6 weeks in the lungs of vaccinated $m$ ice (13), where elimination of challenge parasite mostly occurs (14). We have also demonstrated that IFN $\gamma$ has a critical role in this model of immunity, since protection can be abrogated by up to $90 \%$ following in vivo administration of monoclonal antibody to IFN $\gamma(15)$, and $m$ ice with a disrupted IFN $\gamma$ receptor gene fail to establish optimum levels of resistance to challenge (16).

The role of IL-12 in immunity induced by the attenuated schistosome vaccine

Since cells of the Thl phenotype appear to be so important to the expression of im$m$ unity to the live schistosome vaccine, it $m$ ight be predicted that IL-12 would play a critical role during the development of the i $m m$ une response. We in York have analysed the expression of $m \mathrm{RNA}$ for both the p35 and p40 subunits of IL-12 in the skin exposure site, draining LN and lungs at various times after vaccination. Upregulation of the p40 subunit occurs about day 2 in the LN, coincident with peak expression of IFN $\gamma$ and IL-2 (17). Expression of p40 $m$ RNA is also upregulated in the lungs of vaccinated $m$ ice (17) and appears to be related to the peak of cell infiltration and IFN $\gamma$ production by day 21 (13). Similarly, Wynn et al. (18) reported a steady increase in the expression of p40 $m$ RNA in the lungs of vaccinated $m$ ice, which by day 28 was significantly above the level recorded in the lungs of $m$ ice infected with normal parasites.

Detection of $m$ RNA for IL-12 is suggestive of a role for this cytokine in the protective Th1 response. However, in order to demonstrate a direct role for the biologi- cally active heterodimer, we have administered antibodies against the $\mathrm{p} 70$ heterodimer to vaccinated $m$ ice and assessed para $m$ eters of the immune response and protection (Anderson S, Wilson RA and Mountford AP, unpublished results). Either sheep antiIL-12 (Dr. S. Wolf, Genetics Institute, Cambridge, MA) or rabbit anti-IL-12 antibodies (raised in York) were administered at various time points between days 0 and 15 after exposure of $m$ ice to irradiated cercariae. It appears that the antibody treat $m$ ents only had a $m$ arginal effect $o$ n reducing the level of resistance induced by the irradiated vaccine but this effect was greater when the antibody was given around the time of vaccination rather than once the Th1 immune response had established. The treatments did, however, significantly reduce the ratio of IFN $\gamma:$ IL-4 secretion by LN cells at both days 5 and 15 post-vaccination, thereby showing that IL-12 is involved in the regulation of the Th responses in this model. However, cytokine ablation experiments of this kind are always difficult to interpret since we do not know whether removal of the biologically active molecule has been complete due to the administration of insufficient $o$ inappropriate antibody, or dosage $o$ ver a non-optimum time course. The use of $m$ ice genetically deficient for either the p35 or p40 molecules (5) would overcome this proble $m$ and is now a feasible option.

We have compared the immune responses of IL-12 p40-deficient (KO) mice and their C57BL/6 wild-type controls following vaccination with irradiated cercariae (Anderson $S$, Shires VL, Wilson RA and Mountford AP, unpublished results). Cultured LN cells from $\mathrm{KO} m$ ice had $m$ arkedly altered cytokine profiles with significantly decreased production of IFN $\gamma$ but increased IL-4. This pattern was reflected in the levels of cytokine $m$ RNA in both the LN and lungs after vaccination. Correspondingly, the KO mice had decreased levels of Th1-associated antibodies, but enhanced levels of IgE. Following 
exposure of vaccinated $m$ ice to challenge larvae, cells recovered by lavage from the lungs of KO mice secreted abundant IL-4 and IL- 5 but little IFN $\gamma$. Flow cytometric analysis of these cells recorded a very high proportion of eosinophils. Furthermore, foci around challenge parasites in $\mathrm{KO}$ animals contained large numbers of eosinophils and giant cells. Thus, in the absence of IL-12 the Th1 response normally detected in vaccinated $m$ ice fails to develop and, as a consequence, the levels of protection in KO mice were considerably lower (42\%) than in their wild-type counterparts. These data provide conclusive evidence for the involve$m$ ent $o$ IL-12 in immunity induced by the vaccine, but the residual protection obtained reveals that it is not an absolute require$m$ ent. Moreover, the administration of rIL12 to $\mathrm{KO} m$ ice in the first 11 days postvaccination led to a reconstitution of the Th1-type response, exemplified by increased IFN $\gamma$ and decreased IgE production. Treatment of KO mice with rIL-12 also abolished eosinophil influx and restored the level of protective immunity to that detected in wild-type $m$ ice. The observation of pulmonary and antibody immune responses with wild-type characteristics in KO $m$ ice, five weeks after termination of the IL-12 treatment, demonstrates the stability of the protective Th1 response once it has been induced by the action of IL-12.

Antigens from lung-stage schistosomes induce IFN $\gamma$ and IL-12 production

Although the attenuated schistosome vaccine elicits a dominant Th1 lymphocyte population with a probable role for IL-12, it remains unclear which antigens are responsible. We have demonstrated that attenuated lung-stage larvae are efficient stimulators of protection (19) and they release a number of stage-specific proteins (20). Furthermore, antigenic $m$ aterial released be- tween days 6 and 8 by in vitro-cultured lung-stage larvae and the soluble extracts from whole lung-stage larvae induced LN cells from vaccinated $m$ ice to secrete abundant IFN $\gamma$ but little IL-4 upon restimulation in vitro (21). The released antigens were also particularly efficient stimulators of IFN $\gamma$ release by cells recovered from the airways of vaccinated mice. In contrast, antigens from cercariae and skin-stage larvae induced the lowest levels of IFN $\gamma$ but higher levels of IL-4. These results indicate that in vaccinated $m$ ice an overwhel $m$ ing proportion of the lymphocytes responsive to lung-stage antigens had the Th1 phenotype, whilst a greater number of cells with specificities for early antigens had the Th2 or Th0 phenotype. These observations raise the possibility that specific antigenic constituents of the lung-stage antigen preparations have a propensity to activate cells with a Th1 phenotype.

Stimulation of accessory cells to produce IL-12 by constituents of lung-stage parasites could be one explanation for the polarized development of Th1 cells. In initial experiments naive splenocytes, rich in accessory cells, were cultured with various antigen preparations from different stages of parasite development and the presence of IL-12 in the culture supernatant was determined by the subsequent proliferation of human PHA blasts. Such analyses indicated that antigens released from cultured larvae were superior at stimulating IL-12 production compared to soluble ones from sonicated parasites. Moreover, antigens released from lung-stage parasites were better inducers of IL-12 than those released from transforming cercariae. We have also i $m m$ unised $m$ ice with soluble antigens from whole cercariae or lung-stage larvae and analysed the profile of cytokine $m$ RNA in the skin and draining lymph nodes by RTPCR. This approach showed that lung-stage antigens induced a greater upregulation of IFN $\gamma$ and the newly discovered cytokine 
'interferon gamma inducing factor' (IGIF; 22 ), although the expression of the p 40 subunit of IL-12 remained relatively stable compared to naive $m$ ice.

The use of IL-12 as an adjuvant when coadministered with lung-stage antigens

Our evidence for lung-stage antigens being important inducers of protective i $m$ $m$ unity to schistosomes led us to consider immunising $m$ ice directly with a soluble antigen preparation (SLAP) from lung-stage larvae in conjunction with recombinant IL12 as an adjuvant to boost the Th1 immune response (23).

In these studies, immunisation of $m$ ice intradermally with one or two doses of SLAP $(25 \mu \mathrm{g})$ alone elicited a population of antigen-specific CD4+ lymphocytes (day 5 postimmunisation) which secreted IFN $\gamma$, IL-4 and IL-10, indicating a mixed Th1/Th2 or Th0 response. In contrast, cells from $m$ ice immunised with SLAP + IL-12 (1 $\mu \mathrm{g})$ secreted 5 times as $m$ uch IFN $\gamma$ and wereCD4+ cell dependent. IL-4 production in the mice immunised with SLAP + IL-12 was nonexistent, whilst the secretion of IL-10 was reduced by approximately $50 \%$. This indicates that the coadministration of IL-12 with SLAP leads to the induction of an i $m m$ une response entirely dominated by $\mathrm{Th} 1$ lymphocytes. The administration of IL-12 with antigen also had a dram atic effect upon the isotype composition of serum antibodies. Antigen-specific IgG1 and total $\operatorname{IgE}$ antibodies were almost completely inhibited in $m$ ice receiving $S \mathrm{LAP}+\mathrm{IL}-12, m$ irroring the effect on IL-4 which is important in controlling the switching to these isotypes and underlines the inhibitory effect of IL12 on the development of Th2-mediated immune responses. On the other hand, the levels of IgG2a, which are thought to be enhanced by IFN $\gamma$, were not substantially elevated in mice immunised with SLAP +
IL-12 compared to the levels in mice immunised with SLAP alone.

The most i $m$ portant finding of this study (23) was that the delivery of two doses of SLAP + IL-12 induced significant levels (53.4-37.9\%) of protective immunity to challenge infection, whereas vaccination with $S$ LAP alone did not. Failure to achieve higher levels of protection, similar to those achieved with the live vaccine, $m$ ay be due to the lack of the appropriate signals for primed cells to be recruited to the lungs and/or a suboptimum dosing regime for the SLAP + IL-12 vaccine. In this respect, it was demonstrated that administration of IL-12 to mice previously immunised with the live attenuated vaccine could boost the level of protection induced compared to the level in mice exposed to the live vaccine alone (18). Nevertheless, this first report on the use of IL-12 as an adjuvant in conjunction with soluble antigens to stimulate antiworm protective immunity is very encouraging and IL-12 as an adjuvant $m$ ay aid the future development of recombinant or subunit schistosome vaccines.

Does IL-12 act on NK cells or T cells to prime for the development of Th1 immune responses?

In our paper (23), we also sought to determine whether the observed effects of IL-12 on Th lymphocyte development were $o$ on the T cells themselves, or indirectly via IFN $\gamma$ production by IL-12 acting on NK cells. Ablation of NK cells in vivo following administration of anti-NK1.1 monoclonal antibody to mice immunised with SLAP + IL-12 significantly reduced $(64.5 \%)$ the level of proliferation of CD4+ LN cells (day 5 post-immunisation) compared to $\mathrm{co}$ horts treated with control antibody. However, the level of IFN $\gamma$ secreted by cells from $m$ ice treated with anti-NK1.1 was greatly reduced by $o$ ver $97 \%$. These results indicate that the administration of IL-12 in 
this model stimulates NK cells to produce IFN $\gamma$ which then guides Th cell develop$m$ ent, rather than by acting on the Th1 cells directly. However, it is possible that a direct effect on T cells $m$ ay occur at later times and following repeated administration of IL-12.

We have also addressed this question by administering SLAP + IL-12 to mice with a genetic disruption of the IFN $\gamma$ receptor gene (IFN $\gamma \mathrm{R}^{-/-}$); activated NK or T cells from these mice can produce but not respond to IFN $\gamma$. Antigen-specific IFN $\gamma$ production was elevated to a similar extent in both wild-type and IFN $\gamma \mathrm{R}^{-/-}$animals treated with SLAP + IL-12. This implies that IL-12 (or other IL12-induced cytokines) can directly stimulate lymphocytes with a Th1 phenotype without the requirement for IFN $\gamma$. This corroborates studies using schistosome eggs + IL-12 in

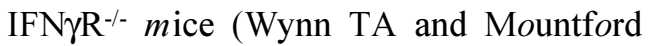
AP, unpublished data) and IFN $\gamma$ knockout $m$ ice (24). It is hard to reconcile the different conclusions from the studies investigating the role of IL-12 in Th1 cell differentiation. Many $o$ ther factors are undoubtedly involved and care $m$ ust be taken when comparing the results obtained from different inbred strains or transgenic animals.

\section{Conclusions}

From a number of studies, it is clear that IL-12 is involved in the regulation of the immune response to schistosomes. It would see $m$ that it does have an important role in the protective Th1 immune response induced by the attenuated vaccine. However, it would be unethical to use such a vaccine in $m$ an and the search for a recombinant or subunit formulation continues. From our studies on lung-stage antigens, which appear to be efficient stimulators of IFN $\gamma$ and IL- 12 production, we have shown that IL-12 can be used as an adjuvant in conjunction with soluble antigens to enhance Th1 immune responses and to induce protection against reinfection. Combined with its role in downregulating Th2 responses associated with pathology, IL-12 represents a powerful tool with which to $m$ anipulate the immune syste $m$ and its use as an adjuvant should aid the development of new and existing candidate vaccine antigens against schistosomes.

\section{References}

1. Wolf SF, Sieburth D \& Sypeck J (1994). Interleukin 12: A key modulator of immune function. Stem Cells, 12: 154-168.

2. Trinchieri G (1995). Interleukin-12: A proinflammatory cytokine with immunoregulatory functions that bridge innate resistance and antigen-specific adaptive immunity. Annual Review of Immunology, 13: $251-276$

3. Sypeck JP, Chung CL, Mayor SEH, Subramanyam JM, Goldman SJ, Sieburth DS, Wolf SF \& Schaub FG (1993). Resolution of cutaneous leishmaniasis: Interleukin 12 initiates a protective $T$ helper type 1 immune response. Journal of Experimental Medicine, 177: 1797-1802.

4. Heinzel FP, Schoenhaut DS, Rerko RM, Rosser LE \& Gately MK (1993). Recombinant interleukin 12 cures mice infected with Leishmania major. Journal of Experimental Medicine, 177: 1505-1509.
5. Mattner F, Magram J, Ferrante J, Launois P, Di Padova K, Behin R, Gately MK, Louis JA \& Alber G (1996). Genetically resistant mice lacking interleukin-12 are susceptible to infection with Leishmania major and mount a polarized Th2 cell response. European Journal of Immunology, 26: 1553-1559.

6. Gazzinelli RT, Hieny S, Wynn TA, Wolf SF \& Sher A (1993). Interleukin 12 is required for the T-lymphocyte-independent induction of interferon $\gamma$ by an intracellular parasite and induces resistance in T-cell-deficient hosts. Proceedings of the National Academy of Sciences, USA, 90: 61156119

7. Finkelman FD, Madden KB, Cheever AW, Katona IM, Morris SC, Gately MK, Hubbard BR, Gause WC \& Urban JF (1994). Effects of interleukin 12 on immune responses and host protection in mice infected with intestinal nematode parasites. Journal of Experimental Medicine, 179: 1563-1572.

8. Pearlman E, Heinzel FP, Hazlett Jr FE \& Kazura JW (1995). IL-12 modulation of T helper responses to the filarial helminth, Brugia malayi. Journal of Immunology, 154: 4658-4664.

9. Wynn TA, Cheever AW, Jankovic D, Poindexter RW, Caspar P, Lewis FA \& Sher A (1995). An IL-12 based vaccination method for preventing fibrosis induced by schistosome infection. Nature, 376: 594596.

10. Pearce EJ, Caspar P, Gryzch J-M, Lewis FA \& Sher A (1991). Downregulation of Th1 cytokine production accompanies induction of Th2 responses by a parasitic helminth, Schistosoma mansoni. Journal of Experimental Medicine, 173: 159-166.

11. Wilson RA (1990). Leaky livers, portal 
shunting and immunity to schistosomes. Parasitology Today, 6: 354-358.

12. Mountford AP, Coulson PS, Pemberton RM, Smythies LE \& Wilson RA (1992). The generation of interferon-gamma-secreting $T$ lymphocytes in the skin-draining lymph nodes and their recruitment to the lungs is associated with protective immunity to Schistosoma mansoni. Immunology, 75: 250-256.

13. Smythies LE, Pemberton RM, Coulson PS, Mountford AP \& Wilson RA (1992). T cell-derived cytokines associated with pulmonary immune mechanisms in mice vaccinated with irradiated cercariae of Schistosoma mansoni. Journal of Immunology, 148: 1512-1518.

14. Smythies LE, Betts C, Coulson PS, Dowling M-A \& Wilson RA (1996). Kinetics and mechanism of effector focus formation in the lungs of mice vaccinated with irradiated cercariae of Schistosoma mansoni. Parasite Immunology, 18: 359369.

15. Smythies LE, Coulson PS \& Wilson RA (1992). Monoclonal antibody to IFN- $\gamma$ modifies pulmonary inflammatory responses and abrogates immunity to Schistosoma mansoni in mice previously vacci- nated with attenuated cercariae. Journal of Immunology, 149: 3654-3658.

16. Wilson RA, Coulson PS, Betts C, Dowling M-A \& Smythies LE (1996). Impaired immunity and altered pulmonary responses in mice with a disrupted interferon- $\gamma$ receptor gene exposed to the irradiated Schistosoma mansoni vaccine. Immunology, 87: 275-282.

17. Betts CJ (1996). RT-PCR analysis of cytokine expression in murine lymph node and lung tissue following exposure to the irradiated Schistosoma mansoni vaccine. Doctoral thesis, The University of York, York, UK.

18. Wynn TA, Jankovic D, Hieny S, Cheever AW \& Sher A (1995). IL-12 enhances vaccine-induced immunity to Schistosoma mansoni in mice and decreases Thelper 2 cytokine expression, IgE production, and tissue eosinophilia. Journal of Immunology, 154: 4701-4709.

19. Coulson PS \& Mountford AP (1989). Fate of attenuated schistosomula administered by different routes, relative to the immunity induced against Schistosoma mansoni. Parasitology, 99: 39-45.

20. Harrop R \& Wilson RA (1993). Protein synthesis and release by cultured schisto- somula of Schistosoma mansoni. Parasitology, 107: 265-274.

21. Mountford AP, Harrop R \& Wilson RA (1995). Antigens derived from lung-stage larvae of Schistosoma mansoni are efficient stimulators of proliferation and gamma interferon secretion by lymphocytes from mice vaccinated with attenuated larvae. Infection and Immunity, 63: 1980-1986.

22. Okamura $H$, Tsutsui $H$, Komatsu $T$, Yutsudo $M$, Hakura A, Tanimoto $T$, Torigoe K, Okura T, Nukada Y, Hattori K, Akita K, Namba M, Tanabe F, Konishi K, Fukada S \& Kurimoto M (1995). Cloning of a new cytokine that induces IFN $\gamma$ production by T-cells. Nature, 378: 88-89.

23. Mountford AP, Anderson $S \&$ Wilson RA (1996). Induction of Th1 cell-mediated protective immunity to Schistosoma mansoni by co-administration of larval antigens and IL-12 as an adjuvant. Journal of Immunology, 156: 4739-4745.

24. Wynn TA, Jankovic D, Hieny S, Zioncheck K, Jardieu P, Cheever AW \& Sher A (1995). IL-12 exacerbates rather than suppresses Th2-dependent pathology in the absence of endogenous IFN $\mathrm{F}$. Journal of Immunology, 154: 3999-4009. 